\title{
Study on oblique shock-induced micro-jet ejection from a grooved metal surface
}

\author{
Y. N. Shi, Q. Zhao, P. Wang, Q. J. Feng \& C. S. Qin \\ Institute of Applied Physics and Computational Mathematics, \\ Beijing, China
}

\begin{abstract}
This work investigates the material ejection from grooved aluminum surfaces in the specific loading conditions associated to oblique shockwaves: one of the shock directions is perpendicular to metal free surface, and another is parallel to the surface. The effects of shock direction as well as groove angle on ejecta production and size distribution are explored. The time evolution of the micro-jet and morphology are simulated using an elastic-plastic hydrodynamic LagrangianRemapping Two-step Eulerian method. A new jet breakup model is developed to predict the dynamic fragmentation of micro-jet and the formation of ejecta. The calculated results show that the micro-jet mass accumulation and necking are due to the velocity gradients along the jet stretching direction, which is dependent on the shock direction. For the z-direction head-on shock, the spatial distribution of jet velocity is a linear acceleration near the groove, and the total ejecta mass increases more significantly than another case. In our simulations, micro-jet mass keeps an increase with the groove angle, and the ejecta particle size is micron. The results compare well with the Sorenson's experiment ones. It is also found that the steady ejecta distributions exhibit a power law scaling with exponents. However, it shows a dramatic difference in the distribution shapes with the shock direction and groove angle variation.

Keywords: micro-jet, oblique shock loading, L-R Two-step Eulerian method, ejection model, ejecta size distribution.
\end{abstract}

\section{Introduction}

An interesting effect in shock-wave phenomena is the behaviour of surfaces shocked to high pressures such as hundreds of kilobars. When a shock wave 
propagating in a solid sample reflects from a free surface, rapid ejection of fine fragments usually happens [1]. It is an extremely complicated and challenging subject in shock physics. This process has been extensively studied both experimentally and theoretically, as a major safety issue for applications such as pyrotechnics, armour design, or inertial confinement fusion, etc.

Previous studies have shown that the formation of ejecta is very complicated, and ejecta are often caused by various factors, such as shock condition, surface microstructure and release melting, etc. [2-4]. There inevitably exist many grooves on metal surfaces in the form of pits, scratches and machine marks. The micro-jet from those grooves may become one kind of main ejection modes. Up to now, significant efforts have been dedicated to studying the micro-jet mechanism. Experiment techniques include thin foils, Asay windows, piezoelectric pins and pulse $x$-rays. The effects of groove shape, shock wave risetime and shock pressure on micro-jet are investigated, including continuum level and molecular dynamics simulations $[5,6]$. Recently, some measured results on ejecta particle size distributions are given [7], which are also predicted by percolation theory and MD simulations. It is shown that the mass, size, and velocity of the ejecta vary depending closely on surface status, initial shock conditions and material properties. Due to the complexities, it is still very difficult to develop a physical-based ejection model and predict the characteristics of ejecta, including the total amount and the size distribution.

In this work, we investigate the micro-jet ejecting process in the specific loading conditions associated to oblique shockwaves: one of the shock directions is perpendicular to metal free surface, and another is parallel to the surface. The time evolution of micro-jet and morphology are simulated using an elastic-plastic hydrodynamic Lagrangian-Remapping Two-step Eulerian method. A new jet breakup model is developed to predict the dynamic fragmentation of micro-jet and the formation of ejecta. The effects of shock direction as well as groove angle on the ejecta production and size distribution are discussed.

\section{Methodology}

The simulation sample is a metal aluminum with a wedged groove of depth $h$ and vertex angle $\theta$ on the free surface along the $z$-axis direction (as shown in Fig. 1). The two specific cases of shock loading directions are considered. One is along the $z$-axis which is perpendicular to metal free surface, and another is along the $R$ axis which is parallel to the surface.

Shock waves are generated by assigning the flyer plate an initial velocity to impact the target. The length of the flyer plate is much longer than that of the target to delay the effects of release fan originated from the free surface of the flyer plate. A proper propagation distance is set between the flyer plate and the groove tip to ensure the formation of a supported shock wave before it encounters the tip. Here, the impact velocity is $3.4 \mathrm{~km} / \mathrm{s}$, and the impact pressure of AL targets is about $30 \mathrm{GPa}$. Geometry parameters of AL targets and shock loading parameters are presented in Table 1. 


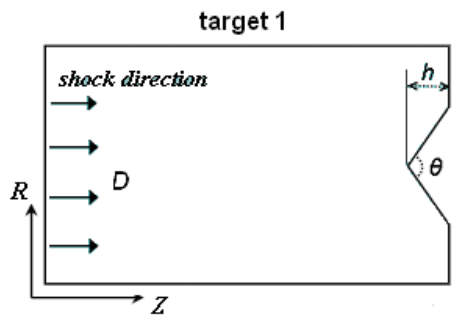

(a) Case-1

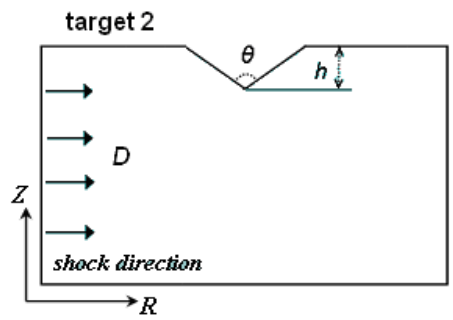

(b) Case-2

Figure 1: Initial configurations of AL target, shock direction: (a) along the $z$-axis, (b) along the $R$-axis.

The elastic-plastic hydrodynamic Lagrangian-Remapping Two-step Euler method is used for the simulation of aluminium micro-jet ejection. The hydrocode uses dimensional split algorithm with Lagrangian re-map and is 2nd order accurate in both time and space. A Young's or VOF approach is employed to capture the interface. The strengths of the code lie in its ability to capture large scale material deformation, wave propagation and interaction generated from impact phenomena. It has been validated extensively against shape charge experiments in which the flow fields are similar to the jetting that is observed in the mass ejection process. It allows for use of different equations of state, strength models and flow field diagnostics, and is specifically designed for the analysis of high strain-rate compressible flow.

Table 1: The computational parameters of the grooved aluminium target.

\begin{tabular}{|c|c|c|c|c|}
\hline Case & Shock direction & Impact pressure & Groove width $(\mu \mathrm{m})$ & Groove angle (deg.) \\
\hline 1 & along $z$-axis & $30 \mathrm{GPa}$ & $20 \cdot \operatorname{tg} 30$ & $30,45,60,90$ \\
\hline 2 & along $R$-axis & $30 \mathrm{GPa}$ & $20 \cdot \operatorname{tg} 30$ & $30,45,60,90$ \\
\hline
\end{tabular}

Here, for the aluminium material, the plastic stress-strain relation is modelled by the von Misses yield criterion, which can be written in terms of the deviator stresses as

$$
S_{r r}^{2}+S_{z z}^{2}+S_{\theta \theta}^{2}+2 S_{r z}^{2} \leq(2 / 3) \sigma_{Y}^{2}
$$

The yield strength of the material $\sigma_{\mathrm{Y}}$ is determined by the Johnson - Cook (JC) model, which is expressed as

$$
\sigma_{Y}=\left[A+B\left(\varepsilon^{p}\right)^{n}\right] \cdot\left[1+C \ln \dot{\varepsilon}^{*}\right] \cdot\left[1-\left(T^{*}\right)^{m}\right],
$$

where $\varepsilon^{\mathrm{p}}$ is the equivalent plastic strain, $\dot{\varepsilon}^{*}=\dot{\varepsilon}^{p} / \dot{\varepsilon}_{0}, \dot{\varepsilon}^{p}$ is the equivalent plastic strain-rate, $\dot{\varepsilon}_{0}=1.0 \mathrm{~s}^{-1}, T^{*}=\left(T-T_{\mathrm{r}}\right) /\left(T_{\mathrm{m}}-T_{\mathrm{r}}\right), T_{\mathrm{m}}$ is the melting temperature and $T_{\mathrm{r}}$ is the room temperature. Parameters $A, B, C, m$ and $n$ are determined by 
experiments. The equation of state for the metal aluminium is the Mie-Gruneisen (MG) equation, which can be described as

$$
\begin{gathered}
p=\frac{\rho_{0} c_{0}^{2} \mu\left[1+\left(1-\frac{\gamma_{0}}{2}\right) \mu-\frac{a}{2} \mu^{2}\right]}{\left[1-\left(S_{1}-1\right) \mu-S_{2} \frac{\mu^{2}}{\mu+1}-S_{3} \frac{\mu^{3}}{(\mu+1)^{2}}\right]^{2}}+\left(\gamma_{0}+a \mu\right) E, \rho \geq \rho_{0} \\
p=\rho_{0} c^{2} \mu+\left(\gamma_{0}+a \mu\right) E, \quad \rho<\rho_{0}
\end{gathered}
$$

where $\mu=\rho / \rho_{0}-1, \rho_{0}$ is the initial density, $c_{0}$ is the initial sound velocity, and $a, \gamma_{0}$ are the Gruneisen coefficients. $S_{1}, S_{2}$ and $S_{3}$ are the parameters that relate the shock speed to the particle velocity, and $E$ is the initial internal energy. A new ejection model coupling with various factors is developed to predict the dynamic fragmentation of micro-jet and the formation of ejecta. The introduction on this model is detailed in Ref. [8]. Here, we just present the coupling motion equation of a single jet, whose expression is

$$
\begin{aligned}
\rho A \frac{\partial^{2} u_{z}}{\partial t^{2}}-\frac{\rho}{8 \pi} & \frac{\partial}{\partial z}\left[\frac{\partial^{2} u_{z}}{\partial t^{2} \partial z}-3 A^{2}\left(\frac{\partial^{2} u_{z}}{\partial t \partial z}\right)^{2}\right]=\frac{\partial\left(A \sigma_{z}\right)}{\partial z} \\
& +\frac{1}{4 \pi} \frac{\partial^{2}}{\partial z^{2}}\left(\frac{A^{2} \sigma_{z R}}{R}\right)+\left[\pi \frac{\partial\left(R F_{T}\right)}{\partial z}+\frac{\partial^{2}}{\partial z^{2}}\left(A F_{T} \frac{\partial R}{\partial z}\right)\right], \\
& +\frac{3}{2} \frac{\partial\left(A Q_{z z}\right)}{\partial z}+\frac{1}{4 \pi} \frac{\partial^{2}}{\partial z^{2}}\left(\frac{A^{2} Q_{z R}}{R}\right)-A \frac{\partial F_{g}}{\partial z}
\end{aligned}
$$

In Eqn (5), $u_{z}, R$ and $A$ are the axial-direction velocity, radius and section area of jet, $\sigma_{\mathrm{z}}$ and $\sigma_{\mathrm{zR}}$ are axial and shear stress, $Q_{\mathrm{zz}}$ and $Q_{\mathrm{zR}}$ are axial and shear viscosity stress, $F_{\mathrm{T}}$ is the surface tension, $F_{\mathrm{g}}$ is gas resistance. Due to stretching and increased necking cause fragmentation of a jet, the formula of the size and number of fragments can be given as follows:

$$
D_{\text {ejecta }}=\sqrt[3]{6 \lambda_{m}} \cdot R, \quad N_{\text {number }}=\int_{V_{0}}^{V_{L}} d V / \Delta V_{z p}
$$

where $\lambda_{\mathrm{m}}$ is a critical wavelength of jet surface disturbance, $\Delta V_{\mathrm{zp}}$ is the jet headtail velocity difference. 


\section{Results and discussion}

\subsection{Mechanisms of jetting}

With the above model and methods, we first carry out the simulations of micro-jet using a case- 1 run with $h=20 \mu \mathrm{m}, \theta=60^{\circ}$ and shock direction along the $z$-axis, to illustrate jetting mechanisms. When a shock wave breaks out at a groove tip, a series of rarefaction waves propagate into the target interior. The matters near the groove free surface acquire $R$-component velocities and move toward the axis of symmetry, as shown in Fig. 2(a). Consequently, the matters above and below the axis collide around the axis, along with the central, fast, forward-moving matters, forming a high speed jet along the shock direction. The main features governing wave reflection from the groove can be approached by shock wave theory. Fig. 2(b) is the two-dimensional description of the interaction of a planar shock wave (shock pressure $P_{1}$, shock velocity $U_{\mathrm{s}}$, particle velocity $V_{1}$ ) with a planar free surface at an incidence angle $\sigma$.

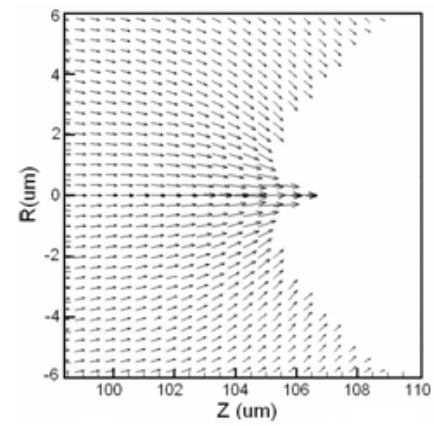

(a)

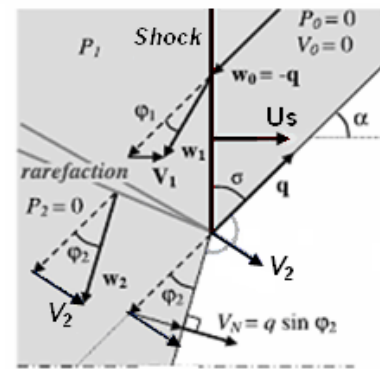

(b)

Figure 2: (a) Velocity vector plot of AL target-1 near the tip of the groove at $=19.7 \mathrm{~ns}$, case- $1, h=20 \mu \mathrm{m}, \theta=60^{\circ}$, and (b) Two-dimensional analytical description in the steady-state regular case.

In the regular case, the shocked state is below the sonic point, and the flow is supersonic, which allows steady-state conditions where velocities and angles remain constant during the interaction. By neglecting pressure decay during the interaction of the planar shock with the groove edge, the resulting jet velocity can be estimated [9]. In Fig. 2(b), $q=U_{\mathrm{s}} / \sin \sigma$ is the shock apparent velocity along the interface, $\alpha=90^{\circ}-\sigma$ is the groove half-angle, $\varphi_{2}$ is the deviation angle after full release and $V_{2}$ is the collapse velocity along the bisecting direction, which can be derived from its normal component.

\subsection{Shock direction dependences of surface jetting}

To investigate the effects of shock direction on surface jetting in more detail, we perform $2 \mathrm{D}$ binning analyses. A spatial distribution of the jet velocity $\left(u\right.$ or $\left.u_{\mathrm{z}}\right)$ is 
plotted in Fig. 3, showing acceleration near the groove, reduced acceleration further away, and deceleration within the jet head. For the case of z-direction headon shock, the acceleration is close to linear, and $u_{\mathrm{z}}$ is symmetric about the axis of symmetry, while $V_{\mathrm{R}}$ shows negative gradients along the z-axis near the groove tip (Fig. 4(a)). While the velocity of jet under oblique shock is lower and its direction is not along the loading direction ( $R$-axis). There lies a deviation angle between the jet velocity $u$ and the $R$-direction (Fig. 4(b)), and the change of the acceleration is nonlinear.

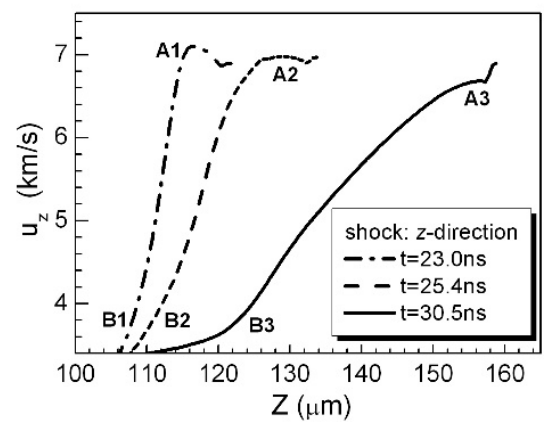

(a)

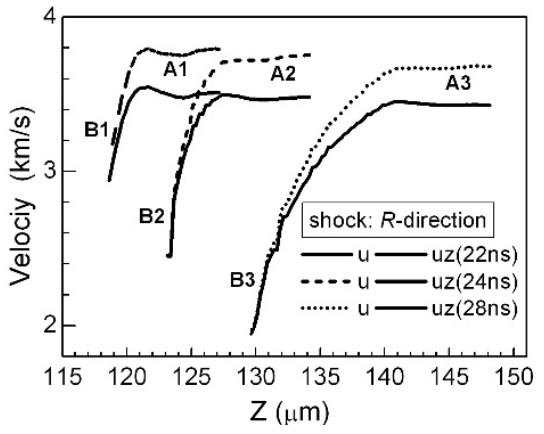

(b)

Figure 3: Spatial distribution of jet velocity. (a) Case-1, head-on shock, along the $z$-direction; (b) Case-2, oblique shock, along the $R$-direction.
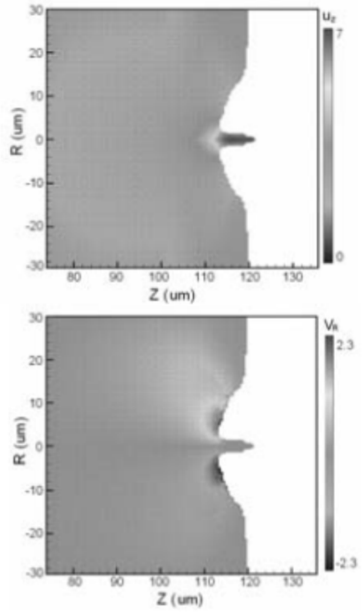

(a) Case-1
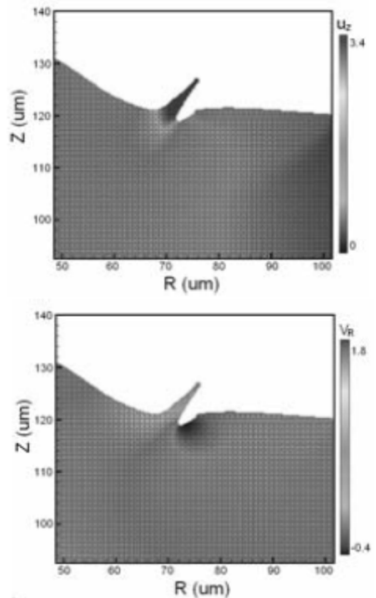

(b) Case-2

Figure 4: 2D maps of velocities $u_{z}$ and $V_{\mathrm{R}}$ (in $\mathrm{km} / \mathrm{s}$ ) on the $R-z$ plane. (a) the $z$ direction shock, at $\mathrm{t}=19.7 \mathrm{~ns}$, (b) the $R$-direction shock, at $\mathrm{t}=23.0 \mathrm{~ns}$. 
Fig. 5 is simulation results of the micro-jet morphology evolution under the above two specific shock conditions. It shows the growth in lateral and longitudinal dimensions followed by necking. The former is accompanied by pronounced mass accumulation, and then, the necking is the dominant phenomenon. Both mass accumulation and necking are due to the velocity gradients along jet stretching direction, as seen in Fig. 3: the gradient values in jet different region give rise to two competing phenomena: mass accumulation in the head (A) and necking (B) behind it. The decrease in their amplitudes as increasing time leads to reduced mass accumulation and increased necking at later times, and necking may even cause fragmentation of a jet.

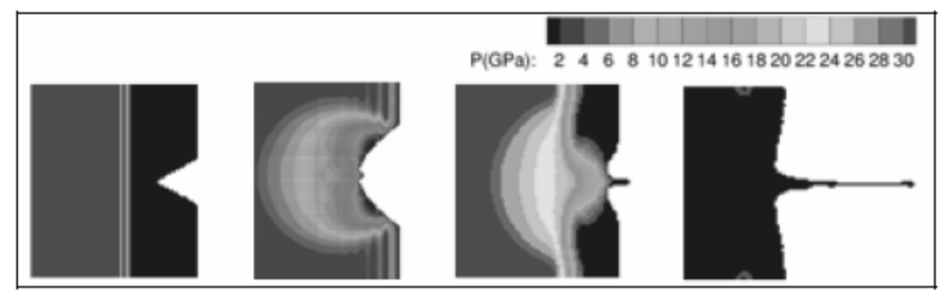

(a) Case-1: the $z$-direction shock.
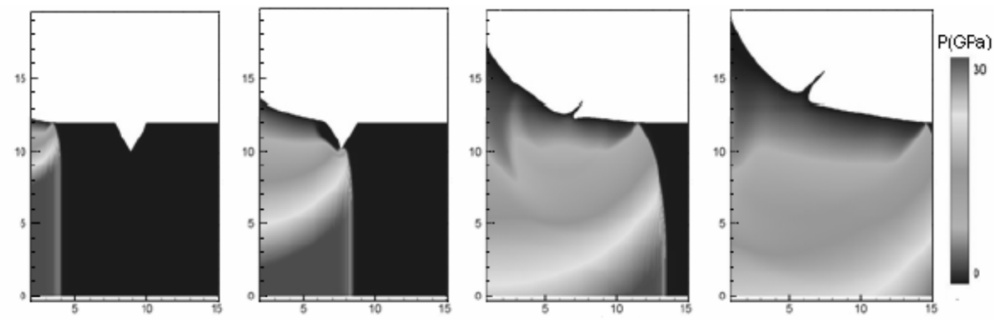

(b) Case-2: the $R$-direction shock

Figure 5: Morphology evolution of micro-jet formation with $h=20 \mu \mathrm{m}, \theta=60^{\circ}$.

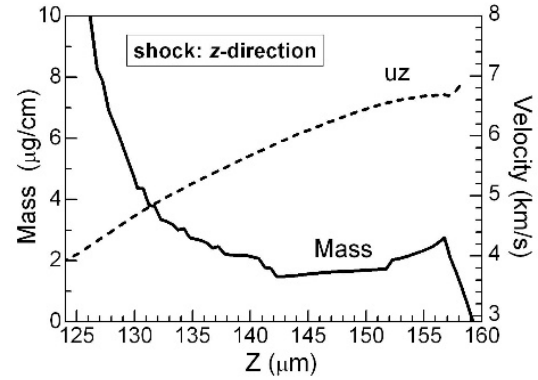

(a)

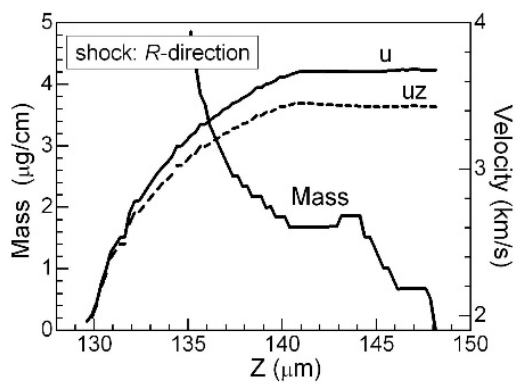

(b)

Figure 6: Effects of shock direction on ejecta mass and jet velocity. (a) $t=30 \mathrm{~ns}$, the $z$-direction shock; (b) $t=28 \mathrm{~ns}$, the $R$-direction shock. 
Fig. 6 compares the mass and velocity distribution of micro-jets with the same impact velocity. It indicates that the jetting is stronger under the $z$-direction headon shock, and the total ejecta mass increases more significantly. Here, due to stretching and increased necking cause fragmentation, the above model (5-6) is used to predict the dynamic process of micro-jet fragmentation. The mass-size distribution of ejecta particles is plotted in Fig. 7. Horizontal ordinate denotes the particle diameter, and vertical ordinate denotes the ratio of ejecta particle mass to the total jetting mass. It is shown that the size of ejecta particles is micron, and the smaller ejecta particles are possessed of more mass percentage. The difference between these distributions is dependent on the shock direction.

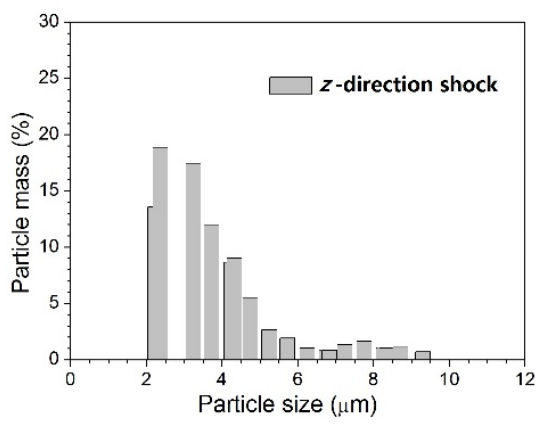

(a)

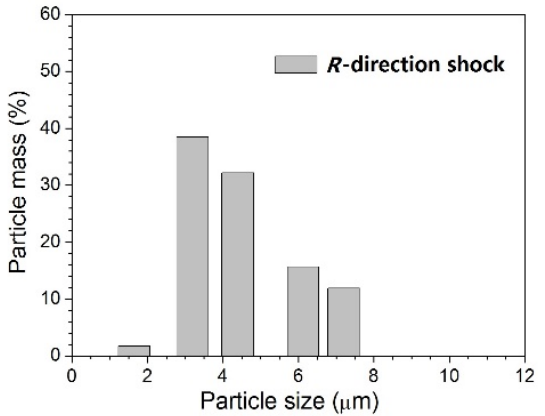

(b)

Figure 7: Mass-size distribution of jetting particles for the case of the $z$-direction shock (a) and $R$-direction shock (b), respectively.

\subsection{Groove angle dependences of surface jetting}

Here, we also conducted some simulations to investigate the effects of groove angle on surface jetting. Fig. 8 compares jet head velocity evolutions for the same width and different angles. It can be seen that with increasing angle, the maximum

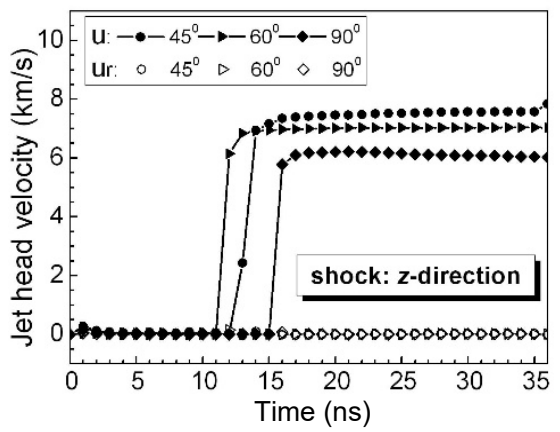

(a)

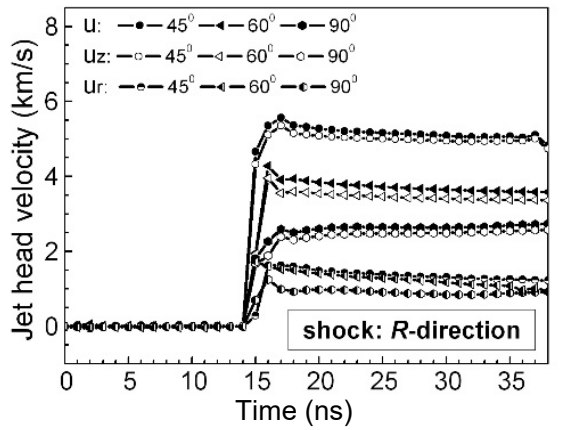

(b)

Figure 8: Jet head velocity $\left(u, u_{\mathrm{z}}\right.$ and $\left.V_{\mathrm{R}}\right)$ evolutions for different groove angles. (a) the $z$-direction shock and (b) the $R$-direction shock. 
and asymptotic values of jet velocity $\left(u, u_{\mathrm{z}}\right)$ decrease. The reason is that, when the groove width is equal, at smaller angles, $u$ is larger and the groove depth is larger along the $z$-axis so the jet head gains more acceleration. They are not necessarily along the axis of symmetry under different shock conditions.

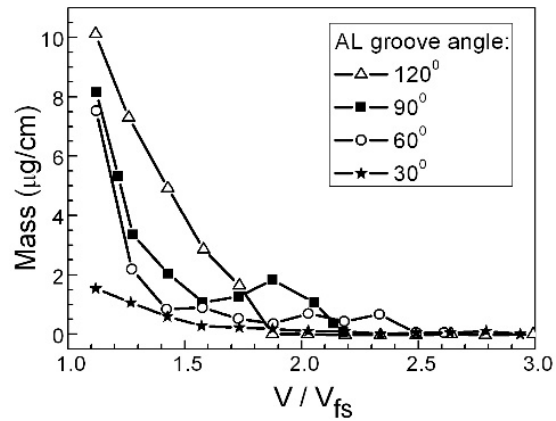

(a)

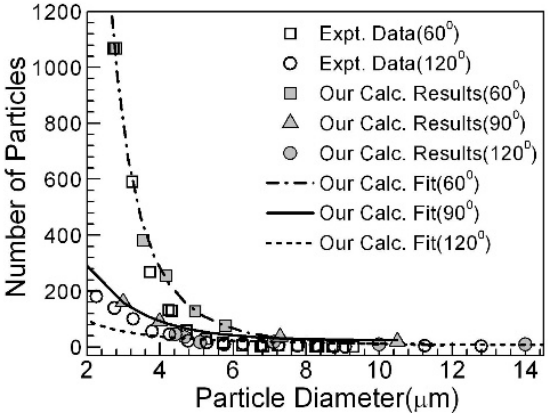

(b)

Figure 9: Mass-velocity distribution (a) and ejecta particle number-size distribution (b) for the $z$-direction head-on shock.

In Fig. 9(a), we also present the mass-velocity distribution for the same depth and different angles. The shock direction is along the z-axis direction and the angle varies between $30^{\circ}$ and $120^{\circ}$ at an increment of $30^{\circ}$. Here, horizontal ordinate denotes the ratio of jetting velocity to surface velocity. The corresponding particle size distributions are shown in Fig. 9(b). One can see that, ejecta mass keeps an increase with angle and the size distribution exhibits a power-law scaling with exponents, in agree well with Sorenson experiment [7]. However, it also shows a dramatic difference in the distribution shapes with the groove angle variation. This difference may be caused by melting or release melting for different groove angle, while the mechanism will be more complicated for the case of the $R$-direction oblique shock. The implications of these results are being further investigated.

\section{Conclusion}

In this work, we have investigated the head-on and oblique shock-induced microjetting from grooved aluminum surfaces using an elastic-plastic hydrodynamic L-R Two-step Eulerian method. A new ejection model is developed to predict the dynamic process of jet breakup and ejecta production. The variation of micro-jet mass and morphology are obtained. The calculated results show that the micro-jet mass accumulation and necking are due to the velocity gradients along jet stretching direction, which is dependent on the shock direction. The effects of shock direction and groove angle on micro-jet evolution and ejecta size distribution are explored, by performing a variety of numerical simulations. It is found that with increasing angle, micro-jet mass keeps an increase while the maximum values of jet velocity decrease. For the $z$-direction head-on shock, 
the spatial distribution of jet velocity is a linear acceleration near the groove, and the total ejecta mass increases more significantly than another case. These microjets fragmentation giving rise to particle size distributions has been presented here. For different angles of groove, the ejecta particle size is micron, and the ejecta distributions exhibit a power law scaling with exponents. The results are well accorded with Sorenson's experiment ones. In addition, it should be pointed out that for different shock directions and groove angles, the dramatic difference in the distribution shapes may be caused by melting or release melting need to be further studied to explore the ejecta formation mechanism on the jet breakup and ejecta production process.

\section{Acknowledgements}

This work was supported by the National Natural Science Foundation of China (No.11371067) and the Science and Technology Development Foundation of China Academy of Engineering Physics (No. 2015B0201044).

\section{References}

[1] Asay, J.R., Material ejection from shock-loaded free surfaces of aluminum and lead. Report No. SAND-76-0542, 1976.

[2] Han, C. S., Jing, F.Q., Ding, J., et al., Study on the phenomena of the mass ejection from the free surface of aluminium at different dynamic loading rates. Chinese J of High Pressure Physics, 3, pp. 97-106, 1989.

[3] Asay, J.R., Effect of shockwave rise-time on material ejection from aluminium surface. Report No. SAND-77-0731, 1977.

[4] Zellner, M.B., McNeil, W.V., Hammerberg, J.E., et al., Probing the underlying physics of ejecta production from shocked Sn samples. Journal of Applied Physics, 103, pp. 123502, 2008.

[5] Dimonte, G., Terrones, G., Cherne, F.J. \& Ramaprabhu, P., Ejecta source model based on the nonlinear Richtmyer-Meshkov instability. Journal of Applied Physics, 113, pp. 024905, 2013.

[6] Durand, O., \& Soulard, L., Large-scale molecular dynamics study of jet breakup and ejecta production from shock-loaded copper with a hybrid method. Journal of Applied Physics, 111, pp. 044901, 2012.

[7] Sorenson, D.S., Minich, R.W., Romero, J.L., Tunnell, T.W. \& Malone, R.M., Ejecta particle size distributions for shock loaded Sn and AL Metals. Journal of Applied Physics, 92(10), pp. 5830-5836, 2002.

[8] Shi, Y.N. \& Qin, C.S., Instability and breakup of stretching metallic jets. Chinese J of Theoretical and Applied Mechanics, 41(3), pp. 361-369, 2009.

[9] Walters, W.P., Fundamentals of shaped charges. High Velocity Impact Dynamics, eds. Zukas J.A., John Wiley \& Sons, New York, pp. 731-829, 1990. 